\title{
Factores asociados con enfermedades no transmisibles en el personal docente $y$ administrativo de una institución universitaria
}

\author{
Factors associated with non-transmissible diseases among academic and administrative personnel in a \\ Peruvian university
}

\author{
Kattia Ochoa-Vigo ${ }^{1, a}$, Carmen Rosa Alvarado-Zúñiga ${ }^{1, b}$, Mónica Graciela del Carmen \\ Mendoza-Ramírez ${ }^{1, c}$, Lesly Estefany Roca-Mauricio ${ }^{1, \mathrm{c}}$
}

\section{RESUMEN}

Objetivo: Determinar la asociación entre los factores de riesgo para enfermedades no trasmisibles comportamentales y metabólicos con algunas variables demográficas y comorbilidades en personal docente y administrativo de una institución universitaria. Material y métodos: Estudio descriptivo, de asociación con diseño transversal. La investigación se realizó en los locales de la Universidad (Campus Universitario y Sede Administrativa-ciudad), según programación, entre los meses de diciembre 2018 a febrero de 2019, con una muestra de 150 participantes elegidos aleatoriamente. Se aplicó test de $\mathrm{Chi}^{2}$, prueba exacta de Fisher y se determinó Odd ratio (OR) e IC al 95\%; se consideró significativo $\mathrm{p} \leq 0,05$. Resultados: El 21,3\% de participantes tenía alguna enfermedad, 11,3\% fumaba y $25,3 \%$ consumía bebida alcohólica; el $66,7 \%$ consumía verduras/hortalizas inadecuadamente, $82 \%$ realizaba deficiente actividad física y $87,3 \%$ tenía dislipidemia; además, el 10,7\% presentó hipertensión arterial, 46,7\% sobrepeso y $27,3 \%$ obesidad. Los factores asociados con hipertensión arterial fueron ser varón (OR=3,59, IC 95\%: 1,10-11,70), edad $\geq 40$ años ( $\mathrm{OR}=4,21$, IC 95\%: 1,15-15,44), casado (OR=3,06, IC 95\%: 1,01-9,31), enfermedad previa $(\mathrm{OR}=6,21$, IC 95\%: 2,09-18,36) y consumir alcohol (OR=4,66, IC 95\%:1,60-13,57). Para la obesidad, ser varón $(\mathrm{OR}=4,54$, IC 95\%:1,97-10,46), residir fuera de Barranca $(\mathrm{OR}=3,12$, IC 95\%: 1,27-7,68) y triglicéridos elevados (OR=10,48, IC 95\%: 1,37-80,33). Conclusiones: Los factores asociados con enfermedad no transmisible en los trabajadores de la Universidad fueron la deficiente actividad física, el bajo consumo de frutas y verduras, el consumo de tabaco y alcohol; y están asociados con los metabólicos y comorbilidades como la hipertensión arterial, obesidad, dislipidemia.

PALABRAS CLAVE: Enfermedades no transmisibles, factores de riesgo, docentes, personal administrativo. (Fuente: DeCS BIREME).

\footnotetext{
Universidad Nacional de Barranca. Barranca, Lima, Perú.

Doctora en Enfermería

Magister en Salud Pública

Estudiante de Iniciación Científica
} 


\section{SUMMARY}

Objective: To investigate for factors associated with non-transmissible diseases and demographic and comorbid conditions among academic and administrative personnel at a Peruvian university. Methods: A cross-sectional study was carried-out at a Peruvian university from December 2018 to February 2019 including 150 participants. The Chi square and Fisher's exact tests were used and odds ratios with $95 \% \mathrm{CI}$ were calculated, a p value $<0,05$ was considered statistically significant. Results: $21.3 \%$ of participants had one comorbidity; $11.3 \%$ were smokers; $25.3 \%$ were drinkers; $66.7 \%$ consumed vegetables regularly; $82 \%$ did not perform physical exercises; $87.3 \%$ had dyslipidemia; $10.7 \%$ had arterial hypertension; $46.7 \%$ were overweight and $27.3 \%$ had obesity. Factors associated with arterial hypertension were male sex $(\mathrm{OR}=3.59$, IC 95\%: 1.10-11.70), age above 40 years $(\mathrm{OR}=4.21$, IC 95\%: 1.15-15.44), married status ( $\mathrm{OR}=3.06$, IC 95\%: 1.01-9.31) and having an underlying disease (OR=6.21, IC 95\%: 2.09-18.36). Factors associated with obesity were, being male (OR=4.54, IC 95\%:1.97-10.46), living outside Barranca (OR=3.12, IC 95\%: 1.27-7.68) and having high triglycerides (OR=10.48, IC 95\%: 1.37-80.33).

Conclusions: Factors associated with non-transmissible diseases were lack of physical exercise, low intake of vegetables and fruits, drinking alcohol and smoking.

KEYWORDS: Non-communicable diseases, risk factors, faculty, administrative personnel. (Source: MeSH NLM).

\section{INTRODUCCIÓN}

Las enfermedades no trasmisibles (ENT) son padecimientos de larga duración y lenta progresión. Entre ellas, destacan las enfermedades cardiovasculares, cáncer, diabetes mellitus y las respiratorias crónicas, con gran impacto en los países en vías de desarrollo, que agravan la problemática socioeconómica y deterioran más la calidad de vida de las poblaciones pobres y vulnerables ${ }^{(1)}$, quienes registran más del $75 \%$ de estas enfermedades y, cada vez, afectan más a personas entre los 30-69 años de edad, con incremento de la mortalidad prematura ${ }^{(2)}$.

Según la Organización Mundial de la Salud (OMS), entre los factores de riesgo para ENT destacan los factores comportamentales modificables y los metabólicos. Considerando como factor comportamental al tabaquismo, la exposición al humo ajeno, la ingesta de alimentos con elevado sodio, consumo de alcohol y la deficiente actividad física ${ }^{(3)}$. Los factores metabólicos son la hipertensión arterial, el sobrepeso y obesidad, hiperglicemia e hiperlipidemia ${ }^{(4)}$. Estudios de base poblacional muestran que los varones tienen más sobrepeso y obesidad que las mujeres, aumentando esta condición en los grupos de mayor edad, bien como en aquellos con menor escolaridad ${ }^{(5,6)}$, bajo ingreso económico ${ }^{(5)}$ y entre los casados/con pareja ${ }^{(6)}$. Además, un tercio de la población padece diabetes o hipertensión ${ }^{(6,7)}$ y casi un $20 \%$ presenta ambas enfermedades (6), hipertrigliceridemia, consumo de tabaco y alcohol ${ }^{(7)}$; con actividad física deficiente ${ }^{(6,7)}$.

La dislipidemia suele estar asociada con la hipertensión arterial, diabetes o tabaquismo ${ }^{(8)}$. Hernández et al. ${ }^{(9)}$, refieren que el colesterol total, c-HDL, c-LDL y los triglicéridos aumentan a mayor edad; adicionalmente, el colesterol total ajustado a la edad se asocia con cualquier factor de riesgo cardiovascular y, más del $70 \%$ de la población tiene al menos un factor cardiovascular. El sobrepeso y el sedentarismo están presentes en la mayoría de personas $(5,6,8,10,11)$, con bajo consumo de frutas y verduras ${ }^{(10)}$.

La amenaza que constituye las ENT en cualquier grupo social, especialmente en el Perú, que atraviesa una post transición epidemiológica desde finales del siglo XX, donde las ENT predominan sobre las demás enfermedades, dado el impacto negativo de la globalización, la urbanización descontrolada y los estilos de vida, con alto consumo de productos hipercalóricos, de bajo valor nutricional, ricos en sal, grasas saturadas, junto al sedentarismo ${ }^{(12)}$.

Uno de los principales problemas es el sistema sanitario deficiente en salud pública, a pesar de las políticas y normas reguladoras vigentes ${ }^{(14)}$ en concordancia con la OMS; además de la escasa o nula información sobre las ENT en los trabajadores ${ }^{(13)}$. 
Bajo este contexto, la universidad es un espacio donde estudiantes, docentes y administrativos pasan largas horas, muchas veces sin oportunidad de retornar al hogar por las distancias geográficas existentes entre un lugar y otro. Docentes y administrativos laboran entre 8-12 horas continuas, interfiriendo con los hábitos de alimentación y prácticas de actividad física, observándose entre los trabajadores sobrepeso y obesidad con posibles enfermedades crónicos degenerativos en la salud ${ }^{(15)}$.

El objetivo del estudio fue determinar la asociación entre los factores de riesgo para ENT comportamentales (el tabaquismo, la exposición al humo, la ingesta de alimentos con elevado sodio, consumo de alcohol y la deficiente actividad física) y metabólicos (hipertensión arterial, el sobrepeso, obesidad, hiperglucemia, hiperlipidemia) con algunas variables demográficas (sexo, edad, residencia, estado civil) y comorbilidades (dislipidemia, hipertensión arterial, diabetes mellitus) en personal docente y administrativo de una institución universitaria.

\section{MATERIAL Y MÉTODOS}

Estudio cuantitativo, descriptivo y de asociación. La población estuvo conformada por los docentes y el personal administrativo que laboraban en una institución universitaria, localizada en el interior de la Región Lima. El estudio fue realizado durante el semestre 2018-II, con una población de 249 (docente $162+$ administrativo 87). Se estimó una muestra utilizando la fórmula de población finita para estudios de proporción, con un nivel de confianza de $95 \%$, un error de 5\% y una probabilidad de acierto de $40 \%$ $(\mathrm{n}=150)$, elegidos mediante muestreo probabilístico, con reposición. Se consideraron como criterios de inclusión: ser personal docente o administrativo de la universidad, ambos sexos, residentes en el país en los últimos cinco años y participación voluntaria. Fueron excluidas las mujeres gestantes.

Se realizó la toma de muestra de sangre venosa en ayunas para determinar glucosa, perfil lipídico (HDL colesterol, LDL colesterol, colesterol total y triglicéridos) medido en el laboratorio utilizándose el método enzimático colorimétrico, la muestra de sangre obtenida fue de $5 \mathrm{ml}$ en tubos al vacío, sin anticoagulante.

La presión arterial fue medida por el encuestador, con el trabajador en posición sentada luego de 10 a 20 minutos de reposo, utilizándose un tensiómetro o esfingomanómetro aneroide con brazalete velcro de marca Riester ${ }^{\circledR}$ con manguito de tamaño medio y un estetoscopio de aluminio también de marca Riester ${ }^{\circledR}$ para todos los participantes.

Para el índice de masa corporal (IMC) se tomaron las medidas de peso y talla utilizando un tallímetro adherido a la pared y una balanza digital marca ADE® Germany, con una capacidad máxima de 150,00 kg.

Se aplicaron dos cuestionarios mediante la entrevista. Uno elaborado a partir del STEPS Instruments propuesto por la OMS para medir factores de riesgo de ENT ${ }^{(16)}$, considerando el cuestionario básico que tiene cinco componentes, la primera parte registra datos del entrevistador y entrevistado, siguen datos del entrevistado y luego 21 criterios sobre: consumo de tabaco, de alcohol y del régimen alimentario.

El segundo instrumento fue el Cuestionario Internacional de Actividad Física-IPAQ. Zhang-Xu et al. ${ }^{(17)}$, citando a Graig, refieren que tiene buena confiabilidad y validez internacional, habiendo sido utilizado en poblaciones de 15-69 años de edad para medir el grado de actividad física. El instrumento fue aplicado en diferentes países ${ }^{(18)}$, incluyendo el Perú ${ }^{(17)}$. Está estructurado en cuatro componentes: tiempo libre, mantenimiento del hogar, ocupacionales y transporte ${ }^{(19)}$; utilizándose en el estudio la versión corta $^{(20)}$.

La actividad física se midió en equivalentes metabólicos (MET). La clasificación se realizó teniendo en cuenta la actividad física de cada participante. Un MET es la energía consumida mientras se permanece sentado/reposo; el valor MET de una actividad o ejercicio se calcula en relación con el reposo. En caso de ejercicio o actividad vigorosa, el esfuerzo debe ser superior a 6 MET. Como ejemplos se puede citar, que caminar a paso ligero equivaldría a 2,5 MET, caminar a paso muy rápido a 4,5 MET. Son actividades vigorosas el baile aeróbico, montar bicicleta, nadar, jugar tenis, correr, entre otros ${ }^{(21)}$.

Serón et al. ${ }^{(22)}$, citando la guía IPAQ, refieren que los MET son una forma de calcular los requerimientos energéticos y que son múltiplos de la tasa metabólica basal y la unidad utilizada de MET-minuto; se calcula multiplicando el MET correspondiente con el tipo de actividad realizada por minuto ejecutado en un día o una semana ${ }^{(18)}$. 
El tercer instrumento fue una ficha de registro de los datos antropométricos y bioquímicos, consta de 7 criterios, constituido por 12 ítems: Presión arterial, peso/talla, glucosa en ayunas, perfil lipídico y triglicéridos.

Para recolectar los datos se cumplieron con los trámites de autorización. La lista de docentes y administrativos fue obtenida de la oficina de recursos humanos de la universidad; luego se procedió a unificarlo intercalando docentes y administrativos obteniéndose el muestreo en el programa Excel 2010. Cada trabajador fue contactado vía celular y personal. La toma de datos fue realizada en locales de la Universidad (Campus Universitario y Sede Administrativa-ciudad), según programación, entre diciembre 2018 y febrero 2019, solicitándoles asistan con ropa ligera y en ayunas de 12 horas.

El trabajador fue informado sobre el objetivo y procedimientos del estudio, dando respuesta a sus inquietudes. Una vez firmado el consentimiento informado fue realizada la entrevista en una sala libre de ruidos, en un tiempo estimado de 15-20 min. Concluido esta fase, se obtenía la muestra de sangre (de 7-10 ml). Continuando con la medición de la presión arterial siguiendo el protocolo ${ }^{(23)}$; la toma de presión arterial se realizó en ambos brazos y para el estudio se consideró el control del lado izquierdo. Todo el procedimiento ocurrió durante 10-12 minutos; terminando con la antropometría conforme normatividad (24); la balanza era regulada cada 10 participantes.

Para el análisis estadístico, los datos previamente fueron digitados en una base de datos en Programa Excel 2010 utilizando códigos. Luego, fueron importados por el Programa SPSS v. 15,0. Se usó la estadística descriptiva con frecuencias absolutas y relativas. Para determinar asociación de variables categóricas se utilizó el test de Chi-cuadrado, test de Fisher y Odd ratio, considerando un valor estadístico significativo de $\mathrm{p}<0,05$.

El proyecto fue revisado y aprobado por el Comité de Ética de la Universidad Nacional de Barranca, aún

Tabla 1. Descripción de las variables en estudio.

\begin{tabular}{|c|c|}
\hline Variables & Categorías \\
\hline Edad & $<40$ años; $\geq 40$ años \\
\hline Sexo & Hombres; Mujeres \\
\hline Residencia & Barranca; Externo a Barranca \\
\hline Estado civil & Soltero; Casado \\
\hline Dislipidemia & $\begin{array}{l}\text { No (colesterol total }<200 \mathrm{mg} / \mathrm{dl} \text {, cLDL }<100 \mathrm{mg} / \mathrm{dl} \text {, cHDL }>40 \mathrm{mg} / \mathrm{dl} \\
\text { y triglicéridos }<150 \mathrm{mg} / \mathrm{dl} \text { ); } \\
\mathrm{Si}(\mathrm{cHDL} \text { disminuido, colesterol total, cLDL, y triglicéridos aumentados) }\end{array}$ \\
\hline Hipertensión Arterial & $\begin{array}{l}\text { No }(<120 /<80 \mathrm{~mm} \mathrm{Hg} \\
\mathrm{Si}(\geq 120 / \geq 80 \mathrm{~mm} \mathrm{Hg})\end{array}$ \\
\hline Diabetes mellitus & No; Si (mediante diagnóstico médico) \\
\hline Actividad física & $\begin{array}{l}\text { Deficiente (consume }<600 \mathrm{MET} \mathrm{min} / \mathrm{semana} \text { ); } \\
\text { Aceptable-Eficiente (consumo } \geq 600 \mathrm{MET} \mathrm{min} / \text { semana). }\end{array}$ \\
\hline Consumo de frutas adecuado & $\begin{array}{l}\mathrm{Si}(\geq 3 \text { porciones/día) } \\
\text { No (consume }<3 \text { porciones/día) }\end{array}$ \\
\hline Consumo de verduras adecuado & $\begin{array}{l}\mathrm{Si}(\geq 2 \text { porciones/día); } \\
\text { No (consume }<2 \text { porciones/día) }\end{array}$ \\
\hline Tabaquismo & $\begin{array}{l}\text { Sí (fuma al menos } 5 \text { cigarro/día); } \\
\text { No (no fuma) }\end{array}$ \\
\hline Consumo de alcohol frecuente & $\begin{array}{l}\text { Si (consumo por lo menos una vez en los últimos } 30 \text { días); } \\
\text { No (sin consumo de bebida alcohólica) }\end{array}$ \\
\hline Índice masa corporal & $\begin{array}{l}\text { Normal }(\text { de } 18,5-24,9) \\
\text { Sobrepeso }(25,0-29,9) \\
\text { Obesidad }(\geq 30,0)\end{array}$ \\
\hline
\end{tabular}


no acreditado por el Instituto Nacional de Salud por ser una universidad recién creada. (Registro $\mathrm{N}^{\circ} 002-$ 2019-UNAB/CEPI).

\section{RESULTADOS}

El $51,3 \%$ era mujer, el $54,0 \% \geq 40$ años con una media y desviación estándar de 41,3 $\pm 11,7,65,3 \%$ residía en Barranca, $44,7 \%$ era casado, 35,3\% trabajaba en otro lugar y $73,3 \%$ pertenecía a familias con menos de cuatro miembros. La IMC media fue $28,0 \pm 4,4$.

En la tabla 2, se muestra la frecuencia de hábitos nocivos, estilo de vida y comorbilidades en la población estudiada; resalta el bajo consumo de verduras y hortalizas en el $66,7 \%$, la actividad física deficiente en el $82,0 \%$ y la dislipidemia en $87,3 \%$.

En la tabla 3, se muestra el nivel de asociación de los factores demográficos y de los factores de riesgo para ENT con la hipertensión arterial en la población estudiada; ser varón, mayor de 40 años, estar casado, padecer de alguna enfermedad y consumir alcohol de manera frecuente, se asociaron con hipertensión arterial.
En la tabla 4, se muestra la asociación de los factores demográficos y de riesgo para ENT con la obesidad y sobrepeso en la población estudiada; ser varón y residir fuera de Barranca se asociaron con la obesidad y sobrepeso.

No se encontró asociación significativa entre los factores demográficos y factores de riesgo para ENT con la dislipidemia (tabla 5), ni con la diabetes (tabla $6)$.

\section{DISCUSIÓN}

Estudios realizados en Uruguay y Tailandia con trabajadores de un banco y una empresa petrolera también muestran mayor presencia de mujeres ${ }^{(24,11)}$, la edad media en trabajadores del banco fue 48 años ${ }^{(24)} \mathrm{y}$ mayores de 30 años en la empresa petrolera ${ }^{(11)}$.

Sobre los factores de riesgo comportamentales para ENT se evidencia alta frecuencia (tabla 2). Aunque el hábito de fumar y consumo de alcohol fueron más bajos que los datos reportados por el INEI a nivel nacional el $2018^{(25)}$. Por otro lado, Skapino y Alvarez (26) identificaron que el $16,1 \%$ fumaba y $49,3 \%$ tenía historia de fumar; la baja frecuencia está relacionado

Tabla 2. Características de estilo de vida, hábitos nocivos y comorbilidades en la población estudiada. Universidad de Barranca-Perú, enero 2019 ( $\mathrm{n}=150)$.

\begin{tabular}{lrr}
\hline Característica & n & \% \\
\hline Fuma actualmente & 17 & 11,3 \\
Consumo frecuente bebida alcohólica & 38 & 25,3 \\
Bajo consumo de frutas & 41 & 27,3 \\
Bajo consumo de verduras y Hortalizas & 100 & 66,7 \\
Actividad Física deficiente & 123 & 82,0 \\
Colesterol- LDL incrementado & 84 & 56,0 \\
Colesterol-HDL incrementado & 72 & 48,0 \\
Colesterol Total incrementado & 38 & 25,3 \\
Triglicéridos incrementados & 25 & 16,7 \\
Hipertensión arterial & 16 & 10,7 \\
Diabetes mellitus & 4 & 2,7 \\
Dislipidemia & 131 & 87,3 \\
Índice de masa corporal (IMC) & & \\
$\quad$ Sobrepeso & 70 & 46,7 \\
Obesidad (IMC $\geq 30)$ & 41 & 27,3 \\
\hline
\end{tabular}


Tabla 3. Asociación de factores demográficos y factores de riesgo para ENT, con hipertensión arterial. Universidad Nacional de Barranca-Perú, enero 2019.

\begin{tabular}{lccccc}
\hline \multirow{2}{*}{ Factor } & \multicolumn{2}{c}{ Hipertensión (\%) } & \multirow{2}{*}{ OR } & IC 95\% & p \\
\cline { 2 - 3 } & Con & Sin & & & \\
\cline { 2 - 3 } Sexo Masculino & $12(75,0)$ & $61(45,5)$ & 3,59 & $1,10-11,70$ & 0,050 \\
Edad $\geq 40$ años & $13(81,25)$ & $68(50,7)$ & 4,21 & $1,15-15,44$ & 0,041 \\
Residencia (Externo a Barranca) & $6(37,5)$ & $46(34,3)$ & 1,15 & $0,39-3,36$ & 1,000 \\
Estado civil Casado & $11(68,8)$ & $56(41,8)$ & 3,06 & $1,01-9,31$ & 0,070 \\
Enfermedades & $9(56,25)$ & $23(17,2)$ & 6,21 & $2,09-18,36$ & 0,000 \\
Tabaquismo & $1(6,25)$ & $16(11,9)$ & 0,49 & $0,06-3,98$ & $0,701^{*}$ \\
Ingesta de alcohol frecuente & $9(56,25)$ & $29(21,6)$ & 4,66 & $1,60-13,57$ & $0,011^{*}$ \\
Ingesta inadecuada frutas, jugos, zumos & $5(31,25)$ & $36(26,9)$ & 1,24 & $0,40-3,81$ & $0,771^{*}$ \\
Ingesta inadecuada verduras u hortalizas & $9(56,25)$ & $91(67,9)$ & 0,61 & $0,21-1,74$ & $0,511^{*}$ \\
Actividad física deficiente & $12(75,0)$ & $111(82,8)$ & 0,62 & $0,18-2,10$ & $0,941^{*}$ \\
\hline
\end{tabular}

*Test de Fisher

Tabla 4. Asociación de los factores demográficos y factores de riesgo para ENT, con obesidad y sobrepeso. Universidad Nacional de Barranca-Perú, enero 2019.

\begin{tabular}{lccccc}
\hline \multirow{2}{*}{ Factor } & \multicolumn{2}{l}{ Sobrepeso / Obesidad (\%) } & \multirow{2}{*}{ OR } & IC 95\% & \multirow{2}{*}{$\mathbf{p}^{*}$} \\
\cline { 2 - 3 } & \multicolumn{1}{c}{ Con } & Sin & & & \\
\cline { 1 - 3 } Sexo Masculino & $64(57,7)$ & $9(23,1)$ & 4,54 & $1,97-10,46$ & 0,00 \\
Edad $\geq 40$ años & $65(58,6)$ & $16(41,0)$ & 2,03 & $0,97-4,26$ & 0,08 \\
Residencia (Externo a Barranca) & $45(40,5)$ & $7(17,9)$ & 3,12 & $1,27-7,68$ & 0,02 \\
Estado civil Casado & $52(46,8)$ & $15(38,5)$ & 1,41 & $0,67-2,97$ & 0,47 \\
Enfermedades & $26(23,4)$ & $6(15,4)$ & 1,68 & $0,64-4,46$ & 0,41 \\
Tabaquismo & $15(13,5)$ & $2(5,1)$ & 2,89 & $0,63-13,26$ & 0,24 \\
Ingesta de alcohol frecuente & $29(26,1)$ & $9(23,1)$ & 1,18 & $0,50-2,78$ & 0,87 \\
Ingesta inadecuada frutas, jugos, zumos & $31(27,9)$ & $10(25,6)$ & 1,12 & $0,49-2,58$ & 0,95 \\
Ingesta inadecuada verduras u hortalizas & $74(66,7)$ & $26(66,7)$ & 1,0 & $0,46-2,17$ & 1,00 \\
Actividad física deficiente & $94(84,7)$ & $29(74,4)$ & 1,91 & $0,7-4,62$ & 0,23 \\
Triglicéridos (valores anormales) & $24(21,6)$ & $1(2,6)$ & 10,48 & $1,37-80,33$ & 0,01 \\
\hline
\end{tabular}

*Test de Fisher

con la política de no fumar de la institución laboral; semejante a la institución en estudio, identificada como universidad saludable libre de tabaco atendiendo a la Ley No29517 (27).

Otro factor fue la inactividad física, siendo mayor que en estudios previos ${ }^{(13,25)}$. Esto muestra que los trabajadores de la universidad tienen escaso tiempo para ejercitarse.
Según la OMS ${ }^{(28)}$, una persona debe caminar con paso ligero al menos 30 min. cinco veces por semana, consumiendo un mínimo de energía de 600 MET-min/ semana para obtener beneficios de la actividad física; caso contrario los riesgos de ENT aumentan.

En el consumo de frutas y verduras, es recomendable consumir cinco porciones de frutas y verduras al día o 400 gramos, siendo esto imposible debido al horario 
Tabla 5. Asociación de factores demográficos y factores de riesgo para ENT con dislipidemia. Universidad Nacional de Barranca-Perú, enero 2019.

\begin{tabular}{lccccc}
\hline \multirow{2}{*}{ Factor } & \multicolumn{2}{c}{ Dislipidemia (\%) } & \multirow{2}{*}{ OR } & IC 95\% & p \\
\cline { 2 - 3 } & Con & Sin & & & \\
\cline { 2 - 5 } Sexo Masculino & $65(49,6)$ & $8(42,1)$ & 1,35 & $0,51-3,58$ & 0,71 \\
Edad $\geq 40$ años & $73(55,7)$ & $8(42,1)$ & 1,7 & $0,65-4,58$ & 0,38 \\
Residencia (Externo a Barranca) & $48(36,6)$ & $4(21,1)$ & 2,17 & $0,68-6,91$ & 0,28 \\
Estado civil Casado & $58(44,3)$ & $9(47,4)$ & 0,88 & $0,34-2,32$ & 0,99 \\
Enfermedades & $28(21,4)$ & $4(21,1)$ & 1,02 & $0,31-3,32$ & $1,00^{*}$ \\
Tabaquismo & $15(11,5)$ & $2(10,5)$ & 1,1 & $0,23-5,23$ & $1,00^{*}$ \\
Ingesta de alcohol frecuente & $34(26,0)$ & $4(21,1)$ & 1,31 & $0,41-4,24$ & $0,781^{*}$ \\
Ingesta inadecuada frutas, jugos, zumos & $38(29,0)$ & $3(15,8)$ & 2,18 & $0,60-7,91$ & 0,35 \\
Ingesta inadecuada verduras u hortalizas & $89(67,9)$ & $11(57,9)$ & 1,54 & $0,58-4,11$ & 0,54 \\
Actividad física deficiente & $110(84,0)$ & $13(68,4)$ & 2,42 & $0,83-7,08$ & $0,111^{*}$ \\
\hline
\end{tabular}

*Test de Fisher

Tabla 6. Asociación de factores demográficos y factores de riesgo para ENT con diabetes mellitus. Universidad Nacional de Barranca-Perú; enero 2019.

\begin{tabular}{lccccc}
\hline \multirow{2}{*}{ Factor } & \multicolumn{2}{c}{ Diabetes (\%) } & OR & IC 95\% & \multirow{2}{*}{$\mathbf{p}^{*}$} \\
\cline { 2 - 3 } & Con & Sin & & & \\
\hline Sexo Masculino & $3(75)$ & $70(47,9)$ & 3,26 & $0,33-32,05$ & 0,36 \\
Edad $\geq 40$ años & $3(75)$ & $78(53,4)$ & 2,62 & $0,27-25,73$ & 0,63 \\
Residencia (Externo a Barranca) & $2(50)$ & $50(34,2)$ & 1,92 & $0,26-14,04$ & 0,61 \\
Estado civil Casado & $4(100)$ & $63(43,2)$ & ND & ND & 0,04 \\
Enfermedades & $2(50)$ & $30(20,5)$ & 3,87 & $0,52-28,59$ & 0,20 \\
Tabaquismo & - & $17(11,6)$ & ND & ND & 0,57 \\
Ingesta de alcohol frecuente & $1(25)$ & $37(25,3)$ & 0,98 & $0,10-9,73$ & 1,00 \\
Ingesta inadecuada frutas, jugos, zumos & - & $41(28,1)$ & ND & ND & 0,58 \\
Ingesta inadecuada verduras u hortalizas & $2(50)$ & $98(67,1)$ & 0,49 & $0,07-3,58$ & 0,60 \\
Actividad física deficiente & $4(100)$ & $119(81,5)$ & ND & ND & 1,00 \\
\hline
\end{tabular}

\section{*Test de Fisher}

$\mathrm{ND}=$ No definido (algunas casillas tienen valor cero)

laboral, reportándose en el estudio más consumo de frutas que verduras y hortalizas. Al respecto, Rosas et al. ${ }^{(16)}$, refieren que el $85,3 \%$ de trabajadores consumía frutas y verduras por debajo de las recomendaciones. La tercera encuesta nacional sobre factores de riesgo para ENT en Argentina reportó un consumo promedio diario de 1,9 frutas o verduras ${ }^{(10)}$.
Entre los factores de riesgos metabólicos para ENT, el sobrepeso y la obesidad en el estudio muestran mayor frecuencia al compararse con información nacional ${ }^{(27)}$, pero semejantes a estudios previos ${ }^{(11,13,25)}$. El sobrepeso y la obesidad constituyen un problema creciente en la población, siendo alarmante y afectando al $40 \%$, y en algunos casos llega a superar el $60 \%{ }^{(11)}$, 
predisponiendo al desenlace de alguna enfermedad cardiovascular, que se potencializa al sumar varios factores de riesgo ${ }^{(29)}$. Hernández et al. ${ }^{(9)}$, refieren que más del $70 \%$ de la población presenta al menos un factor de riesgo cardiovascular.

Además, la frecuencia de hipertensión arterial y de diabetes en la población estudiada fue baja, contrarios a la dislipidemia. Un estudio uruguayo mostró que la hipertensión arterial afectaba al 30,2\% y la dislipidemia al $33,1 \%$ de población ${ }^{(25)}$. La alta frecuencia de dislipidemia en los trabajadores del estudio, triplica los valores obtenidos en otros estudios, siendo un dato importante, sobre todo, por sus consecuencias en la salud, como enfermedades cardiacas incluso, derrame cerebral ${ }^{(30)}$; reconociendo, además, que una proporción de personas con hipertensión y diabetes desconocen que la padecen ${ }^{(25)}$.

En nuestro estudio, se encontró asociación con hipertensión arterial, el ser varón, tener $\geq 40$ años, padecer de alguna enfermedad, y consumir alcohol. Sin embargo, Maguiña ${ }^{(31)}$ en su estudio identifica un total de 33 adultos que presentan una mayor predisposición a desarrollar hipertensión arterial por ser mayores de 40 años, por lo tanto, evidenciamos que la edad es un factor no modificable, que afecta a la población en estudio.

Se encontró asociación con obesidad, el ser varón, y residir fuera de Barranca y tener triglicéridos alterados. Rosas et al. ${ }^{(13)}$, reportan que el colesterol total, cLDL, triglicéridos, cHDL estaban elevados en las personas obesas $(\mathrm{p}<0,05)$ estableciendo asociación entre una ENT como: enfermedad cardiovascular, diabetes, enfermedad respiratoria crónica, cardiometabólicas, hipertensión arterial e hiperlipidemia, con tener $\geq 40$ años de edad y con tener sobrepeso ${ }^{(11)}$.

En el caso de alimentos no fue incluido en el estudio el consumo de grasas trans, bebidas azucaradas y alto contenido de sodio, tomando en consideración previos estudios ${ }^{(11,25,32)}$.

Es importante prevenir enfermedades cardiovasculares en la población estudiada, sobre todo, al considerar que ser varón, mayor de 40 años, con obesidad constituyen factores de riesgo para enfermedades cardiovasculares ${ }^{(30)}$. Rosas et al. ${ }^{(13)}$, citando a Maggio y Pi-Sunyer, refieren que el sobrepeso y la obesidad constituyen riesgos en numerosas dolencias, entre ellas la dislipidemia, que afecta a una alta proporción de trabajadores. Al respecto, pocos estudios a nivel nacional la abordan, a pesar de ser reconocida como predictor de enfermedad cardiaca o derrame cerebral, dato que marca relevancia en las enfermedades discapacitantes, y cada vez más, en poblaciones menores de 60 años ${ }^{(29)}$.

Entre las limitaciones del estudio, resaltar el tamaño de la muestra comparado con estudios previos y su diseño transversal, debiendo corroborarse los resultados con estudios de causa-efecto. Asimismo, pudo estar presente el sesgo de memoria, pues no siempre las acciones cotidianas se recuerdan con precisión. Además, algunos trabajadores no participaron del estudio, posiblemente por la escasa cultura de participar en estudios de investigación. Por último, otra limitación fue el instrumento utilizado para determinar la presión arterial.

Concluyendo, el estudio muestra que en la población estudiada los factores de riesgo para ENT como consumir alcohol, bajo consumo de frutas y verduras, la hipertensión, obesidad y dislipidemia, tienen asociación con el ser varón, tener $\geq 40$ años, padecer de alguna enfermedad; los cuales deben ser considerados al implementar estrategias de prevención y control con eficacia en los centros laborales y, en el caso de la universidad, promover actividades orientados a persuadir y preservar estilos de vida saludable. Actualmente, existe interés por crear ámbitos de esparcimiento y desarrollo de actividades físicas vigorosas con personal capacitado con acceso a la comunidad universitaria y dispendio de alimentos saludables dentro del claustro.

\section{Declaración de financiamiento y de conflictos de intereses:}

Proyecto Financiado por la Universidad Nacional de Barranca.

Los autores no presentan conflictos de interés en la publicación del artículo.

\section{Contribución de autoría:}

KOV: Participó en la concepción y diseño del estudio, análisis e interpretación de los resultados, revisión crítica del artículo y aprobación de la versión a ser publicada. CRAZ: Participó en el diseño del estudio, análisis e interpretación de los resultados, revisión crítica del artículo y aprobación de la versión a ser publicada. MGCMR y LERM: Participó en la adquisición de datos, interpretación de resultados y aprobación de la versión a ser publicada. 


\section{Correspondencia:}

Kattia Ochoa-Vigo

Calle John Neper 104, Dpto. 303

Urbanización San Borja Sur

Lima 41, Lima-Perú.

Correo electrónico: kochoa@unab.edu.pe

Celular: 51995920067

\section{REFERENCIAS BIBLIOGRÁFICAS}

1. Organización Panamericana de la Salud. Enfermedades no transmisibles en las Américas: Construyamos un futuro más saludable 2011. Washington, D.C: Organización Panamericana de la Salud; 2011 (Citado el 15 de mayo 2018). Disponible en: http://www.paho.org/col/index.php?option=com docman view=download\&category slug= publicacions-ops-oms\&alias $=1063$-entamericas 202011\&Itemid $=688$

2. Organización Mundial de la Salud. Enfermedades no trasmisibles. Ginebra: Organización Mundial de la Salud; 2018. (Citado el 10 de julio 2018). Disponible en: http://www.who.int/es/news-room/ fact-sheets/detail/noncommunicable-diseases

3. Lim SS, Vos T, Flaxman AD, et al. A comparative risk assessment of burden of disease an injury attributable to 67 risk factors and risk factor clusters in 21 regions, 1990-2010: a systematic analysis for the Global Burden of Disease Study 2010. Lancet. 2012; 380(9859): 2224-2260. DOI: https://doi.org/10.1016/ S0140-6736(12)61766-8

4. GBD 2015 Risk Factors Collaborators. Global Burden Disease 2015 Risk Factors Collaborators. Global, regional, and national comparative risk assessment of 79 behavioral, environmental and occupational, and metabolic risks or clusters of risks, 1990-2015: a systematic analysis for the Global Burden of Disease Study 2015. Lancet. 2016; 388(10053):1659-1724. DOI: https://doi. org/10.1016/S0140-6736(16)31679-8

5. Brenner D, Poirier AE, Haig TR, Akawung A, Friedenreich CM, Robson P. Measures of excess body weight and anthropometry among adult Albertans: cross-sectional results from Alberta's tomorrow project cohort. BCM Public Health 2017; 17: 899 -900. DOI: https://doi.org/10.1186/s12889$017-4887-2$

6. DiBonaventura MD, Meincke H, Le Lay A, Fournier J, Bakker E, Ehrenreich A. Obesity in Mexico: prevalence, comorbidities, associations with patient outcomes and treatment experiences. Diabetes Metab Syndr Obes. 2017; 11: 1-10. DOI: https://doi. org/10.2147/DMSO.S129247

7. Thakutr JS, Jeet G, Pal A, et al. Profile of risk factors for non-communicable diseases in Punjab, Northern India: results of a State-Wide STEPS Survey. PloS ONE. 2016; 11(7): e0157705. DOI: https://doi. org/10.1371/journal.pone.0157705

8. Oliveira LB, Carvalho BI, Escorcio-Dourado CSM, Dourado JCL, Nascimento MO. Prevalencia de dislipidemias e fatores de risco associados. J Health Biol Sci. 2017; 5(4):320-325. DOI: https://doi. org/10.12662/2317-3076jhbs.v5i4.1306.p320325.2017

9. Hernández E, Vega AAT, Lozano AJE, Álamo SR, Castrodeza SJJ, Lleras MS. Dislipidemias y riesgo cardiovascular en la población de Castilla y León. Gac Sanit. 2010; 24(4):282-287. DOI: http://doi. $\operatorname{rog} / 10.1016 / j$.gaceta.2010.04.004

10. Galante M, Konfino J, Ondarsuhu D, et al. Principales resultados de la tercera encuesta nacional de factores de riesgo de enfermedades no trasmisibles en Argentina. Rev Argent Salud Pública. 2015; 6(24):2229.

11. Jalayondeja C, Jalayondeja W, Mekhora K, Bhuanantanondh P, Dusadi-Isariyavong A, Upiriyasakul R. Break in sedentary behavior reduces the risk of noncommunicable diseases and cardiometabolic risk factors among workers in a petroleum company. Int J Environ Res Public Health. 2017 may 9; 14(5):501. DOI: http://doi.org/10.3390/ ijerph 14050501

12. Ramos W, Venegas D, Honorio H, Pesantes J, Carrasco J, Yahui M. Enfermedades no trasmisibles: efecto de las grandes transiciones y los determinantes sociales. Rev Peru de Epidemiol. 2014; 1: 1-10. (Citado el 10 de julio 2018). Disponible en: http:// www.redalyc.org/pdf/2031/203132677006.pdf

13. Rosas AA, Lama G, Llanos-Zavalaga F, Dunstan YJ. Prevalencia de obesidad e hipercolesterolemia en trabajadores de una institución estatal de Lima-Perú. Rev Peru Med Exp Salud Pública. 2002; 19(2): 8792.

14. Ministerio de Salud. Resolución Ministerial 229-2016/ MINSA. Lineamientos de políticas y estrategias para la prevención y el control de enfermedades no trasmisibles (ENT) 2016-2010. Lima-Perú: Ministerio de Salud; 2016. p.33. (Citado el 10 de julio 2018). Disponible en: ftp://ftp2.minsa.gob.pe/ normaslegales/2016/RM 229-2016-MINSA.pdf.

15. Gómez- Landeros O, Galvan-Amaya G, ArandaRodriguez R, Herrera-Chacon C, Granados Cosme J. Prevalencia de sobrepeso, obesidad y antecedentes de enfermedad crónica en universitarios mexicanos. Rev Med Inst Mex Seguro Soc. 2018; 56(5): 462467. (Citado el: 9 de abril 2020). Disponible en: ht tps://www.redalyc.org/jats Repo/ 4577/457758201006/html/index.html

16. World Health Organization. Noncommunicable diseasses and their risk factors. The STEPS 
Instrument and Support Material. Ginebra: World Health Organization; 2018 (Citado el 10 de julio 2019). Disponible en: http://www.who.int/ncds/ surveillance/steps/instrument/en

17. Zhang-Xu A, Vivanco M, Zapata F, Málaga G, Loza C. Actividad física global de pacientes con riesgo cardiovascular aplicando el International Physical Activity Questionnaire (IPAQ). Rev Med Hered. 2011; 22(3): 115-120. (Citado el 10 de julio 2018). Disponible en: http://www.scielo.org.pe/pdf/ $\mathrm{rmh} / \mathrm{v} 22 \mathrm{n} 3 / \mathrm{v} 22 \mathrm{n} 3 \mathrm{ao} 4 . \mathrm{pdf}$

18. Mantilla TSC, Gómez-Conesa A. El cuestionario internacional de actividad física. Un instrumento adecuado en el seguimiento de la actividad física poblacional. Rev Iberoam Fisioter Kinesiol. 2007; 10(1):48-52. (Citado el 10 de julio 2018). Disponible en: http://www.redalyc.org/pdf/170/17049838010. pdf

19. IPAQ Group. International Physical Activity Questionnaire. s.n.: IPAQ; 2002 (Citado el 10 de julio 2018). Disponible en: https://sites.google.com/ site/theipaq

20. IPAQ Group. International Physical Activity Questionnaire. Downloadable questionnaires. s.n.: IPAQ; 2002. (Citado el 20 de julio 2018). Disponible en: Disponible en: https://sites.google.com/site/ theipaq/questionnaire_links

21. World Confederation for Physical Therapy. ¿Cómo medir la actividad física? London: WCPT; 2017. (Citado el 30 mayo 2018). Disponible en: https:// www.wcpt.org/sites/wcpt.org/files/files/wptday/17/ Infographics/Spanish/MeasuringPhysicalActivity infographic_A4_FINAL_Spanish_profprint.pdf

22. Serón P, Muñoz S, Lanas F. Nivel de actividad física medida a través del cuestionario internacional de actividad física en población chilena. Rev Med Chile. 2010; 138:1232-1239. (Citado el 20 de julio 2018). Disponible en: https://scielo.conicyt.cl/pdf/ rmc/v138n10/art\%2004.pdf

23. Alba HM. Fundamentos de enfermería. $4^{a}$ ed. Ciudad de México: Editorial Trillas; 2018. pp.336-42.

24. Aranceta BJ. Nutrición comunitaria. $3^{\text {a }}$ ed. Madrid, España: Elservier Masson; 2013. pp.45-52.

25. Instituto Nacional de Estadística e Informática. Enfermedades no transmisibles y no transmisibles, 2017. Lima, Perú: Instituto Nacional de Estadística e Informática; 2018.

26. Skapino E, Alvarez R. Prevalencia de factores de riesgo de enfermedades no transmisibles en funcionarios de una institución bancaria del Uruguay. Rev Urug Cardiol. 2016; 31:246-255. (Citado el 20 de agosto del 2018). Disponible en: http://www. scielo.edu.uy/pdf/ruc/v31n2/v31n2a09.pdf

27. Congreso de la República del Perú. Ley No 29517 Ley que modifica la Ley $\mathrm{N}^{\circ} 28705$, Ley General para la Prevención y Control de los Riesgos del Consumo de Tabaco, para adecuarse al Convenio Marco de la Organización Mundial de la Salud (OMS) para el Control del Tabaco. Lima: Diario Oficial El Peruano; abril 2 de 2010. (Citado el 20 de julio 2018). Disponible en: http://www.digesa.minsa.gob.pe/ norma_consulta/Ley_29517.pdf

28. Organización Mundial de la Salud. Actividad física. Ginebra; Organización Mundial de la Salud; 2018. (Citado el 20 de julio 2018). Disponible en: http:// www.who.int/es/news-room/fact-sheets/detail/ physical-activity

29. Organización Mundial de la Salud. Informe sobre la situación mundial de las enfermedades no trasmisibles. Ginebra: Organización Mundial de la Salud; 2010. (Citado el 20 de julio 2018). Disponible en: https:// www.who.int $/ \mathrm{nmh} /$ publications/ncd_report summary_es.pdf

30. Hormone Health Network. Hiperlipidemia. Washington DC: Hormone Health Network; 2012 (Citado el 20 de julio 2018). Disponible en: https://www.hormone.org/ /media/hormone/files/ spanish/questions-and-answers/heart-health/fs_cmd hyperlipidemia_sp-612.pdf

31. Maguiña PK. Factores de riesgo que predisponen a hipertensión arterial en adultos que acuden al servicio de triaje del Centro de salud Max Arias Schreiber 2013. Lima- Perú. Universidad Nacional Mayor de San Marcos. 2014.pp.50. Disponible en:https://cybertesis. unmsm.edu.pe/bitstream/handle/20.500.12672/3536/ Magui $\%$ C3\%B1a_dp.pdf? sequence $=1 \&$ isAllowed $=y$

32. Randovanovic CAT, Santos LA, Barros Carvalho MD, Marcon SS. Hipertensión arterial y otros factores de riesgo asociados a las enfermedades cardiovasculares en adultos. Rev Latino-Am Enfermagen. 2014; 22(4):547-553. (Citado el 22 de diciembre del2019). Disponible en: http://www.scielo. br/pdf/rlae/v22n4/es_0104-1169-rlae-22-04-00547. pdf

Recibido: 01/05/2020

Aceptado: 28/10/2021 